\title{
RELATIVE FLUORIDE RESPONSE OF CARIES LESIONS CREATED IN FLUOROTIC AND SOUND TEETH STUDIED UNDER REMINERALIZING CONDITIONS
}

Short title: Fluoride response of caries lesions in fluorotic teeth

Hala Alhawij, Frank Lippert, Esperanza Angeles Martinez-Mier*

Indiana University, Department of Preventive and Community Dentistry, Oral Health

Research Institute, 415 Lansing Street, Indianapolis, IN 46202-2876, USA

*Corresponding author:

Esperanza Angeles Martinez-Mier

Indiana University

Department of Preventive and Community Dentistry

Oral Health Research Institute

415 Lansing St

Indianapolis, IN 46202-2876

USA

Phone: +1 (317)-274-8822

E- Mail: esmartin@iu.edu

Keywords: Fluoride, Enamel fluorosis, Dental caries, Demineralization, Remineralization, QLF

This is the author's manuscript of the article published in final edited form as: Alhawij, H., Lippert, F., \& Martinez-Mier, E. A. (2015). Relative fluoride response of caries lesions created in fluorotic and sound teeth studied under remineralizing conditions. Journal of Dentistry, 43(1), 103-109. http://doi.org/10.1016/j.jdent.2014.10.014 


\title{
RELATIVE FLUORIDE RESPONSE OF CARIES LESIONS CREATED IN FLUOROTIC AND SOUND TEETH STUDIED UNDER REMINERALIZING CONDITIONS
}

\begin{abstract}
:
Objectives: The present in vitro $\mathrm{pH}$ cycling study investigated potential differences between caries lesions created in fluorosed and sound enamel with regards to their responsiveness to fluoride under remineralizing conditions.
\end{abstract}

Methods: 360 human first molars (sound and fluorosed) were divided into four groups based on their Thylstrup-Fejerskov score (TF0-3). Each group was further divided into two treatment groups $(\mathrm{n}=45)$ : deionized water or $383 \mathrm{ppm}$ fluoride. Artificial enamel caries lesions were created and $\mathrm{pH}$ cycled for 20 days using an established net remineralization model. Quantitative light-induced fluorescence was used throughout the study to investigate lesion severity and changes thereof. Data were analyzed using two-way ANOVA.

Results: There were no differences in lesion severity between all groups after lesion creation ( lesion $=0.1934$ ). The TF score vs. treatment interaction was significant at all other time points ( $\left.p_{10 d}=0.0280 ; p_{20 d}=<0.0001 ; p_{\text {seddemin }}=0.0411\right)$. Relative differences in responsiveness to fluoride vs. deionized water increased with increasing TF scores. In comparison to lesions created in sound enamel, lesions created in enamel with moderate fluorosis (TF 2/3) were more prone to remineralization in the presence than in the absence of fluoride. Furthermore, lesions created in enamel with moderate fluorosis exhibited more remineralization in the presence of fluoride than lesions created in sound teeth, whereas the opposite was true for deionized water.

Conclusion: Bearing in mind the limitations of laboratory research, the extent of enamel fluorosis severity may directly impact subsequent lesion re- and progression as well as the lesion's responsiveness to fluoride.

Clinical relevance: Caries lesions in fluorotic teeth are more vulnerable to progression but respond more strongly to fluoride than those in non-impacted teeth.

Keywords: Fluoride, Enamel fluorosis, Dental caries, Demineralization, Remineralization, QLF 


\section{Introduction}

Enamel fluorosis is a hypomineralization of dental enamel caused by prolonged ingestion of excessive amounts of fluoride during tooth development. ${ }^{1}$ The severity of enamel fluorosis depends on the amount of fluoride exposure, the age of the child, their individual response, as well as other factors including nutritional status. The association between enamel fluorosis and fluoride exposure has been reported since the early 1900 s. $^{2}$

There is evidence that the prevalence of enamel fluorosis in many countries has increased over the last three decades along with a noted decrease of dental caries. ${ }^{3}$ As a consequence of the reported enamel fluorosis prevalence increase, the U.S. Department of Health and Human Services (HHS) and the U.S. Environmental Protection Agency (EPA) recently changed their recommendations on the optimal level of fluoride in water to maximize the protective effects of fluoride while limiting the occurrence of enamel fluorosis. The level has now been set at $0.7 \mathrm{mg}$ of fluoride per liter of water; instead of the previously recommended range of 0.7 to $1.2 \mathrm{mg} / \mathrm{l}$. The HHS based this changed recommendation on an observed increase in the prevalence of enamel fluorosis as a result of increased fluoride intake from multiple sources.

Fluorosed enamel is characterized by outer hypermineralization and subsurface hypomineralization. The higher concentration of fluoride is believed to affect cell/matrix interactions as the teeth are forming. ${ }^{4}$ Hypomineralization of enamel is caused by the retention of amelogenins in the early maturation stage of tooth development ${ }^{5}$. As a result, the affected enamel does not mature and has surface and subsurface porosities. The tooth becomes more porous than sound teeth, with porosity increasing relative to the severity of fluorosis; the degree and extent of porosity increases in a dose-related manner relative to the tissue/fluid concentrations of fluoride. ${ }^{6}$ 
Very few studies have evaluated the effectiveness of fluoride on lesion progression in fluorosed teeth and compared remineralization patterns between fluorosed and non-fluorosed, sound teeth. Their findings were contradictory; Suma et al. showed that dental caries increased and enamel thickness decreased with increased severity of enamel fluorosis in fluoride endemic areas. ${ }^{7}$ Waidyasekeraet et al. found that moderately fluorosed enamel showed a significant caries resistance. In contrast, mild and moderately fluorosed dentine was significantly more susceptible to caries in vitro. ${ }^{8}$ Driscoll and colleagues found a higher proportion of teeth with severe fluorosis were decayed or filled, and attributed it to pitting of the teeth, staining or both. ${ }^{9}$ On the other hand, a study conducted in the US on schoolchildren showed that molars with moderate-to-severe enamel fluorosis had lower caries prevalence than those without enamel fluorosis. ${ }^{10}$ The chemical, morphological and histologic characteristics of fluorosed teeth may explain the reported variations in caries experience and also partially explain differences between fluorosed and sound enamel with regards to lesion progression and patterns of demineralization and remineralization. However, our understanding of the consequences of enamel fluorosis on lesion formation and remineralization is still poor and deserves further attention by the research community. Furthermore, the extent of enamel fluorosis severity in particular and related consequences on de- and remineralization in the absence or presence of fluoride has not been addressed adequately in the past. Consequently, the aims of the present in vitro study were: 1) to investigate the relative fluoride response of caries lesions created in sound and fluorotic teeth of varying severities of fluorosis under remineralizing conditions in vitro; and 2) to investigate the impact of the presence and severity of enamel fluorosis on caries lesion formation. The null hypothesis for the present study was: There is no significant difference in the 
effectiveness of fluoride to enhance caries lesion remineralization, defined as change in enamel fluorescence relative to lesion baseline, between fluorosed teeth and non-fluorosed, sound teeth.

2. Material and Methods

\subsection{Specimen Collection}

A total of 360 extracted permanent human first molar teeth (90 non-impacted, sound and 270 non-impacted, fluorotic teeth) were divided into four groups ( $\mathrm{n}=90$ in each group, one specimen per tooth) depending on their TF score $(0 ; 1 ; 2 ; 3)$, teeth with visual signs of caries, cracks, extrinsic and/or intrinsic staining were unsuitable for this study and discarded. The extracted teeth were stored in $0.1 \%$ thymol solution (Sigma-Aldrich, MO, USA) until used.

\subsection{Diagnosis of Enamel Fluorosis Severity}

Enamel fluorosis severity in extracted teeth was visually assessed using Thylstrup-Fejerskov Index (TFI) ${ }^{11,12}$ by two examiners. Overall kappa statistic was 0.92 and the weighted kappa was 0.95 that indicates good agreement between the examiners.

\subsection{Specimen preparation}

Before sectioning, all teeth were gently cleaned using a polishing wheel but without abrasive to remove only any debris and other surface contaminants that could potentially interfere with the study aims. Then, the teeth were cut in half coronally-apically and mesially-distally 
leaving buccal and lingual tooth halves from which the roots were removed. As the susceptibility to fluorosis varies between tooth surfaces ${ }^{13}$ and to eliminate surface-specific differences in natural fluoride concentration ${ }^{5}$ which would have been confounding factors, only the buccal half was used in the present study; the lingual half and all other tooth sections were discarded. Subsequently, a sound enamel window, approx. $200 \mu \mathrm{m}$ deep and measuring approx. $3 \times 2 \mathrm{~mm}$, measured by using a digital caliper) was created in the cervical part of each buccal half (using a Sof-Lex disk 3M, MN, US) and slow speed (NSK Nakanishi Inc. Kanuma, Japan). This procedure removed the outer layer that was affected by fluorosis to expose a standardized sound enamel surface. This window served as the sound enamel reference area for QLF measurements. The crown halves were then mounted individually onto one inch square acrylic blocks using non-fluorescent dental impression wax (Alminax, Kemdent, UK) to facilitate repeat QLF measurements. The entire tooth surface, apart from an experimental window, measuring approx. $3 \times 3 \mathrm{~mm}$ in the center of the specimen, and the sound enamel window were covered with red-colored nail varnish (Sally Hansen Advanced, Hard as Nails Nail Polish, NY, USA). The previously created sound enamel window was covered with colorless, clear nail varnish (Sally Hansen Advanced, Hard as Nails Nail Polish, NY, USA). The specimens were balanced into four main groups of 90 specimens each (TF scores $0 ; 1 ; 2 ; 3)$ and each main group was then divided into two subgroups of 45 specimens (fluoride, deionized water/placebo) based on $\Delta \mathrm{F}_{\text {lesion }}$ (see 2.4.).

\subsection{Quantitative Light-induced Fluorescence (QLF)}

All specimens were air-dried for at least 30 min before QLF measurements were performed using the QLF Clinic System and the QLF Patient software v.3.0.0.35 (Inspektor Research, 
the Netherlands). The clear nail varnish used to protect the sound enamel window was not removed, renewed or otherwise altered prior to QLF measurements. Acquired QLF images were analyzed using the QLF Analysis software v.2.00f. $\Delta \mathrm{F}$ was the sole variable and its values were recorded at a threshold level of $5 \%$, i.e. a minimum of $5 \%$ fluorescence loss between sound (i.e. the area covered by clear nail varnish) and the experimental window. The distance between the camera and the surface of the specimen was kept constant throughout the experiment to facilitate repeat measurements.

QLF images were recorded at four time points throughout the study:

a) after specimen preparation $\left(\Delta \mathrm{F}_{\text {base }}\right)$

b) after artificial caries lesion creation $\left(\Delta \mathrm{F}_{\text {lesion }}\right)$

c) after $10 \mathrm{~d}$ of $\mathrm{pH}$ cycling $\left(\Delta \mathrm{F}_{10 \mathrm{~d}}\right)$

d) after $20 \mathrm{~d}$ of $\mathrm{pH}$ cycling; i.e. completion of $\mathrm{pH}$ cycling phase $\left(\Delta \mathrm{F}_{20 \mathrm{~d}}\right.$; primary variable $)$

The primary variable of interest was the difference in $\Delta \mathrm{F}$ values between lesion formation and $20 \mathrm{~d}$ of $\mathrm{pH}$ cycling, $\Delta \Delta \mathrm{F}_{20 \mathrm{~d}}$, which was calculated using the following equation:

$\Delta \Delta \mathrm{F}_{20 \mathrm{~d}} *=\Delta \mathrm{F}_{20 \mathrm{~d}}-\Delta \mathrm{F}_{\text {lesion }}$

*positive $\Delta \Delta \mathrm{F}$ values indicate remineralization, whereas negative $\Delta \Delta \mathrm{F}$ values indicate (further) demineralization.

Other variables of interest were:

$\Delta \Delta \mathrm{F}_{\text {lesion }}=\Delta \mathrm{F}_{\text {lesion }}-\Delta \mathrm{F}_{\text {sound }}$

$\Delta \Delta \mathrm{F}_{10 \mathrm{~d}}=\Delta \mathrm{F}_{10 \mathrm{~d}}-\Delta \mathrm{F}_{\text {lesion }}$ 


\subsection{Artificial Caries Lesion Formation}

Artificial caries lesions were formed in the experimental windows by a 14-day immersion into a solution containing $0.1 \mathrm{M}$ lactic acid; $4.1 \mathrm{mM} \mathrm{CaCl}_{2} \times 2 \mathrm{H}_{2} \mathrm{O} ; 8.0 \mathrm{mM} \mathrm{KH}_{2} \mathrm{PO}_{4}$ (all Fisher Scientific, MA, USA); and 2.0 g/l Carbopol 907 (BF Goodrich Co, Cleveland, OH, USA), pH adjusted to 5.0 using $\mathrm{KOH}$ (Fisher Scientific, MA, USA). Lesion formation was conducted at $37^{\circ} \mathrm{C}$ and using approx. $60 \mathrm{ml}$ demineralization solution per specimen. The solution was renewed after $7 \mathrm{~d}$. After demineralization, specimens were rinsed under running deionized water for at least $1 \mathrm{~min}$. Specimens were then stored at approx. 100\% relative humidity until further use (e.g. QLF measurements).

\subsection{Specimen Balancing Procedure}

Specimens were initially balanced into four main groups of 90 specimens solely based on TF scores $(0 ; 1 ; 2 ; 3)$. Each main group was then divided further into two subgroups of 45 specimens (fluoride, no fluoride/placebo) based on $\Delta \mathrm{F}_{\text {lesion. }}$ Balancing was performed to yield two subgroups within each main group of equal mean $\Delta F_{\text {lesion }}$ values and a sample size of 45 per subgroup.

\section{7. $\quad$ pH Cycling Phase}

The present study utilized a $\mathrm{pH}$ cycling model with a net remineralization outcome that was

loosely based on that presented by White. ${ }^{14,15}$ The daily cycling regimen, which was repeated for $20 \mathrm{~d}$, is shown in Table 1 . 
Artificial saliva (2.20 g/l gastric mucin [American Laboratories Inc., NE, USA], 1.45 mM $\mathrm{CaCl}_{2} \times 2 \mathrm{H}_{2} \mathrm{O}, 5.40 \mathrm{mM} \mathrm{KH}_{2} \mathrm{PO}_{4}, 28.4 \mathrm{mM} \mathrm{NaCl}, 14.9 \mathrm{mM} \mathrm{KCl}$ [all Fisher Scientific, MA, USA], $\mathrm{pH}$ 7.0) was used as the remineralization medium for all groups. Freshly prepared artificial saliva was used each day (changed during the acid challenge period). Treatments were either a $383 \mathrm{ppm}$ F as sodium fluoride (383mg/l) (Fisher Scientific, MA, USA) aqueous solution (fluoride groups; positive control) or deionized water (placebo groups). A $383 \mathrm{ppm} \mathrm{F}$ solution is equivalent in its fluoride concentration to an $1150 \mathrm{ppm} \mathrm{F}$ dentifrice after 1:2 slurry dilution.

\subsection{Statistical Analysis}

Analysis of variance two-way ANOVA was used, followed by pair-wise comparisons using Fisher's Protected Least Significant Differences to control the overall significance level at 5\% to conduct the present study with $n=45$ per subgroup with study power of $80 \%$. The primary variable was $\Delta \Delta \mathrm{F}_{20 \mathrm{~d}}$. A sample size of $\mathrm{n}=45$ per subgroup was chosen to compensate for unexpectedly high biological variation

\section{Results}

The results and statistical analysis for all treatment groups and study variables can be found in Table 2.

All specimens exhibited some fluorescence loss in relation to the created sound enamel reference window after specimen preparation, which, however, was irrespective of their TF 
score $(p=0.799)$. Likewise, fluorosis severity did not affect lesion severity after initial lesion creation $(p=0.193)$.

Enamel fluorosis levels had a significant effect on lesion remineralization after 10 days of $\mathrm{pH}$ cycling ( $p=0.044$; Fig. 1$)$, which was also treatment dependent $(p=0.028)$. Fluoride-treated teeth with TF score 3 showed significantly more remineralization than placebo-treated teeth with TF 3 ( $p=0.036$ ). Teeth with TF scores 0,1 or 2 did not show any fluoride response. Teeth with mild to moderate fluorosis exhibited more remineralization than initially sound teeth.

All treatment groups showed a fluoride response after 20 days of $\mathrm{pH}$ cycling $\left(\Delta \mathrm{F}_{20 \mathrm{~d}}\right.$; primary variable; Fig. 2), with the interaction between TF score and treatment being significant $(p<0.001)$. The fluoride-treated teeth with TF score 2 and 3 had significantly more remineralization than placebo-treated teeth with TF score $2(p=0.0003)$ and $3(p<0.0001)$. Moreover, the fluoride-treated teeth with score $2(p=0.0397)$ and score $3(p=0.0016)$ exhibited significantly more remineralization than fluoride-treated teeth which were initially sound $(\mathrm{TF}=0)$. On the other hand, teeth with score $1(p<0.0001)$ and score $2(p=0.147)$ in the placebo group had more resistance to lesion progression than teeth with score 3 in the same group. To summarize, teeth that had enamel fluorosis with different severity showed more remineralization than sound teeth in the presence of fluoride.

\section{Discussion}

There is little evidence regarding the effectiveness of fluoride on lesion progression in fluorotic teeth, and the comparison between the remineralization patterns of fluorotic teeth and non-fluorotic teeth as mentioned previously (see introduction).The results of the present 
study demonstrated that enamel fluorosis levels have a significant effect on lesion remineralization after $\mathrm{pH}$ cycling. Fluoride was more effective in enhancing caries lesion remineralization in lesions created in fluorosed rather than non-fluorosed, sound teeth. Furthermore, the degree of fluorosis severity at baseline directly impacted the subsequent extent of remineralization.

Artificial caries lesions were created in buccal surface of extracted human first permanent molars (sound and fluorosed), which are more susceptible to fluorosis, also to avoid the effect of the variation of fluoride concentration in different tooth types. ${ }^{13}$ Therefore, the aim of the present study was to study the different responses of early caries lesions created in sound and fluorosed teeth to fluoride under remineralizing conditions.

The net remineralization model proposed by White ${ }^{14,15}$ has been utilized to fulfill the present study's objectives. This model has been used previously and can be considered one of the most suitable models to study the effectiveness of fluoride in remineralizing early caries lesions. Due to the nature of the present study substrate, the present authors decided to employ QLF instead of more conventional assessment techniques, such as transverse microradiography or cross-sectional microhardness. Since QLF provides visual and quantitative feedback, it is suitable for the assessment of de- and remineralization of early enamel lesions as those studied presently and as shown in many previous studies. ${ }^{16,17}$ Moreover, it has an uncomplicated analysis process which also allows for repeat measurements of the same specimen, thereby reducing variability. QLF, however, has limitations due to its inability to differentiate between fluorescence loss as a result of fluorosis and other forms of developmental enamel defects and tooth surface phenomena such as enamel fractures and extrinsic stain on one hand and fluorescence loss due to mineral loss on 
the other. Hence, sound enamel QLF measurements $\left(\Delta \mathrm{F}_{\text {base }}\right)$ were performed in the present study and used in the calculations for all other variables to eliminate QLF specificity issues as a confounding factor. Fluorescent imaging techniques such as QLF have been reported to exhibit a high sensitivity but reduced specificity. ${ }^{18}$ This perhaps explains the presence of fluorescence loss after specimen preparation (i.e. before lesion creation) in the present study, which, however, was irrespective of the TF score (Table 2) and can therefore be excluded as a confounding factor.

In the present study, no significant differences were found between sound teeth and fluorotic teeth after lesion creation $\left(\Delta \Delta \mathrm{F}_{\text {lesion }}\right)$, which may be due to the shallowness of the created lesions combined with a potential lack of sensitivity of the chosen analytical technique QLF. This finding was dissimilar to McInnes et al. who found a negative correlation between the degree of enamel fluorosis and dental caries prevalence. ${ }^{19}$ However, the level of fluoride incorporated into dental mineral by systemic ingestion is insufficient to play a significant role in caries prevention. ${ }^{16}$ Driscoll et al. state that the mild and moderate fluorosed dentine was more susceptible to caries in vitro in the absence of fluoride. ${ }^{9}$

After 10 days of $\mathrm{pH}$ cycling, no significant differences were found between teeth treated with fluoride, although directionally more remineralization was observed in teeth with fluorosis. In placebo-treated teeth, however, only teeth with TF score 3 exhibited further demineralization, whereas all other groups showed some remineralization. Aforementioned differences in structure and porosity can be held accountable for the present observations. Teeth with (severe) fluorosis offer more binding sites for fluoride due to their greater porosity, are, however, more susceptible to further demineralization as acid can diffuse faster and further into the tooth. Nonetheless, differences between treatment groups were rather muted after 10 
days of $\mathrm{pH}$ cycling which may have been too short a period to discern between the effects of fluoride and fluorosis severity.

After 20 days of $\mathrm{pH}$ cycling, differences between treatment groups became more apparent. Fluoride-treated teeth with score 2 and score 3 exhibited significantly more remineralization than placebo-treated teeth of the same scores. Moreover, the fluoride- treated teeth with score 2 and score 3 showed significantly more remineralization than fluoride-treated, sound teeth (i.e. score 0). The degree and extent of porosity increases in a dose-related manner relative to the tissue fluid concentrations of fluoride in teeth. ${ }^{20}$ The teeth with enamel fluorosis have more affinity to fluoride and other minerals, and this may explain the better response of fluorotic teeth. On the other hand, teeth with score 1 and score 2 in the placebo group showed more resistance to lesion progression than teeth with score 3 . This is likely due to hypomineralization of the surface and subsurface enamel of severely fluorotic teeth. A previous study demonstrated that mild and moderately fluorosed dentine was significantly more susceptible to caries in vitro in the absence of fluoride, ${ }^{9}$ highlighting similarities to the present findings on enamel. The chemical, morphological and histological characteristics of fluorotic teeth may explain the reported variations in caries experience and could be linked to differences in response to fluoride effects.

The teeth that had enamel fluorosis with different severity showed more remineralization than sound teeth in the presence of fluoride. However, fluorotic enamel was more prone to further demineralization than sound enamel in the absence of fluoride. This likely is due to the high degree of porosity in fluorosed teeth, which consequently leads to a higher affinity to fluoride than in non-fluorosed teeth. Moreover, fluoride has a high affinity to calcium, which results in (remineralized) enamel of relatively greater acid resistance. ${ }^{16}$ Also, Judith et al. have 
demonstrated that it is possible to increase mineral uptake of fluorosed teeth in vitro by using a supersaturated (with respect to enamel) solution. ${ }^{21}$ In hindsight, investigating tooth histology and morphology would have allowed for a better understanding of the differences in fluoride response of the investigated teeth. However, this will be the focus of future studies.

The present study has some limitations, one of the limitations is the difficulty in the identification of small carious lesions in fluorosed teeth due to the structure of fluorosed teeth. ${ }^{5}$ To overcome this potentially confounding factor, a sound enamel window was created on the teeth close to the cervical area and through abrasion, thereby standardizing all teeth. It is important to realize, however, that fluorosed teeth have a variation in enamel structure even from section to section, as enamel fluorosis appears clearer in cusps and incisal areas. Thus, the use of any part of the tooth as a reference area can still be criticized. Unfortunately, it is not easy to find a definitive solution to this problem, but the central area can be assumed to be the best uniform structure to create a window. QLF imaging appears to have a high sensitivity but reduced specificity when employed in the detection and quantification of fluorosis. An inherent limitation of QLF is the inability to differentiate fluorescence loss as a result of fluorosis; other forms of developmental enamel defects and tooth surface phenomena such as carious lesion, enamel fractures and extrinsic stain. There is evidence to suggest that the use of computer software techniques may facilitate this process; ${ }^{20}$ however this would involve more complicated image processing and tooth mapping prior to analysis. Lastly, the history of the studied teeth prior to extraction was unknown in the present study which may explain the observed variability.

\section{Conclusion}


Considering the limitations of this in vitro study, the data suggest that the extent of enamel fluorosis severity may directly impact the re- and progression as well as the lesion's responsiveness to fluoride. The results of this study will help facilitate the design of future studies on this topic.

References

1. Centers for Disease Control and Prevention. Achievements in Public Health, 19001999: Fluoridation of Drinking Water to Prevent Dental Caries MMWR 1999; 48: 933-940.

2. Black GV, McKay FS. Mottled enamel. An endemic developmental imperfection of the teeth, heretofore unknown in the literature of dentistry. Dental Cosmos 1916; 58: 132 .

3. Kukleva MP, Kondeva VK, Isheva AV, Rimalovska SI. Comparative study of dental caries and dental fluorosis in populations of different dental fluorosis prevalence. Folia Medica (Plovdiv) Journal 2009; 51: 45-52.

4. Denbesten PK, Li W. Chronic fluoride toxicity: dental fluorosis. Monogr Oral Science 2011; 22:81-96.

5. Fejerskov O, Silverstone LM, Melsen B, Møller IJ. Histological features of fluorosed human dental enamel. Caries Research 1975; 9: 190-210. 
6. Allen K, Agosta C, Estafan D. Using microabrasive material to remove fluorosis stains. The Journal of the American Dental Association 2004; 135: 319-23.

7. Suma RL, Shashibhushan KK, Shashikiran ND, Subba Reddy VV. Progression of artificial caries in fluorotic and non-fluorotic enamel. An in vitro study. Journal of Clinical Pediatric Dentistry 2008; 33:127-30.

8. Waidyasekera T, Nikaido D, Weerasinghe DS, Wettasinghe KA, Tagami J. Caries susceptibility of human fluorosed enamel and dentine. Journal of Dentistry 2007; 35: 343-349.

9. Driscoll WS, Horowitz HS, Meyers RJ, Heifetz SB, Kingman A, Zimmerman ER. Prevalence of dental caries and dental fluorosis in areas with negligible, optimal, and above-optimal fluoride concentrations in drinking water. The Journal of the American Dental Association 1986; 113:29-33.

10. Hiroko I, Kumar J. The association between enamel fluorosis and dental caries in US schoolchildren. The Journal of the American Dental Association 2009; 140; 855-62.

11. Thylstrup A, Fejerskov O. Clinical appearance of dental fluorosis in permanent teeth in relation to histological changes. Community Dentistry and Oral Epidemiology $1978 ; 6: 315-28$.

12. Ellwood R, O'Mullane D, Clarkson J, Driscoll W. A comparison of information recorded using the Thylstrup Fejerskov index, Tooth Surface Index of Fluorosis and Developmental Defects of Enamel index. International Dental Journal 1994; 44:62836. 
13. Weerasinghe DS, Nikaido T, Wettasinghe KA, Abayakoon JB,Tagami J. Micro shear bond strength and morphological analysis of self-etching primer adhesive system to fluorosed enamel. Journal of Dentistry 2005; 33:419-426.

14. White DJ. Reactivity of fluoride dentifrices with artificial caries. II. Effects on subsurface lesions: $\mathrm{F}$ uptake, $\mathrm{F}$ distribution, surface hardening and remineralization. Caries Research. 1988; 22:27-36.

15. White DJ. Reactivity of fluoride dentifrices with artificial caries. (Pt 1). Effects on early carious lesions: fluoride uptake, surface hardening and remineralization. Caries Research1987; 21:126-40.

16. Hafström-Björkman U, Sundström F, de Joselin de Jong E, Oliveby A. Comparison of laser fluorescence and longitudinal microradiography for quantitative assessment of in vitro enamel caries. Caries Research 1992; 26:241-7.

17. Lagerweij JM, Damen MD, ten Cate JM. The effect of a fluoridated toothpaste on dentinal lesions in plaque-filled grooves: an intra-oral. Journal of Dental Research 1996; 75: 1687-91.

18. McGrady, M.G., Ellwood, R.P., Taylor, A, et al Evaluating the use of fluorescent imaging for the quantification of dental fluorosis. BMC Oral Health 2012, 12:46-47

19. McInnes PM, Richardson BD, Cleaton-Jones PE. Comparison of dental fluorosis and caries in primary teeth of preschool-children living in arid high and low fluoride villages. Community Dentistry and Oral Epidemiology 1982; 10:182-6. 
20. McGrady MG, Browne D, Ellwood RP, Pretty IA. Differentiation of demarcated enamel opacities from fluorosis using quantitative light-induced fluoroescence. Caries Research 2008, 42:213

21. Judith L, Christensen JL, Larsen MJ, Fejerskov O. Effect of a mineralizing solution on sections of fluorosed human dental enamel in vitro. Caries Research 1979; 13: 47-56. 


\section{FIGURE CAPTIONS OF MANUSCRIPT}

Illustration (1) - Table 1. Treatment schedule for the $\mathrm{pH}$-cycling phase.

Illustration (2) - Table 2. Means (standard deviations) and $p$ values for each factor and their interaction for all treatment groups and study variables

Illustration (3) - The $\Delta \Delta \mathrm{F} 10 \mathrm{~d}$ variable for all placebo groups as a function of enamel fluorosis severity

$\Delta \Delta \mathrm{F} 10 \mathrm{~d}$ : Fluorescence loss after 10 days of $\mathrm{pH}$ cycling

DF: Dental Fluorosis

$0,1,2,3$ : Score of enamel fluorosis severity

F: with Fluoride (treatment group)

No F: Without Fluoride (Placebo group)

$P: p$ Value

Illustration (4) - The $\Delta \Delta \mathrm{F} 20 \mathrm{~d}$ variable for all placebo groups as a function of enamel fluorosis severity

$\Delta \Delta \mathrm{F} 10 \mathrm{~d}$ : Fluorescence loss after 10 days of $\mathrm{pH}$ cycling

DF: Dental Fluorosis

$0,1,2,3$ : Score of enamel fluorosis severity

F: with Fluoride (treatment group)

No F: Without Fluoride (Placebo group)

P: p Value 
Table 1: Treatment schedule for the $\mathrm{pH}$-cycling phase

\begin{tabular}{|l|l|}
\hline Time & pH- cycling phase \\
\hline 1 min & Fluoride/placebo treatment \\
\hline 1 hour & Artificial Saliva \\
\hline 1 min & Fluoride/placebo treatment \\
\hline 1 hour & Artificial Saliva \\
\hline 4 hours & Acid challenge \\
\hline 1 hour & Artificial Saliva \\
\hline 1 min & Fluoride/placebo treatment \\
\hline 1 hour & Artificial Saliva \\
\hline 1 min & Fluoride/placebo treatment \\
\hline (overnight) & Artificial Saliva \\
\hline
\end{tabular}


Table 2. Means (standard deviations) and $p$ values for each factor and their interaction for all treatment groups and study variables

\begin{tabular}{|l|l|l|l|l|l|l|}
\hline $\begin{array}{l}\text { TF } \\
\text { Score }\end{array}$ & Group & $\begin{array}{l}\text { Treatment } \\
\text { during pH } \\
\text { cycling } \\
\text { phase }\end{array}$ & $\begin{array}{l}\Delta \mathrm{F}_{\text {sound }} \\
\text { Means (Sd) }\end{array}$ & $\begin{array}{l}\Delta \Delta \mathrm{F}_{\text {lesion }} \\
\text { Means (Sd) }\end{array}$ & $\begin{array}{l}\Delta \Delta \mathrm{F}_{10 \text { days }} \\
\text { Means (Sd) }\end{array}$ & $\begin{array}{l}\Delta \Delta \mathrm{F}_{20 \text { days }} \\
\text { Means (Sd) }\end{array}$ \\
\hline 0 & $\mathrm{~A}$ & Fluoride & $-12.36(6.1)$ & $-13.14(14.5)$ & $0.07(8.9)$ & $6.19(11.6)$ \\
\hline & $\mathrm{B}$ & No fluoride & $-13.73(5.9)$ & $-14.45(16.1)$ & $0.73(10.1)$ & $5.29(9.8)$ \\
\hline 1 & $\mathrm{C}$ & Fluoride & $-13.17(6.4)$ & $-12.72(12.8)$ & $3.54(9.2)$ & $9.50(13.9)$ \\
\hline & $\mathrm{D}$ & No fluoride & $-13.69(7.0)$ & $-14.49(12.7)$ & $6.27(8.7)$ & $6.21(8.6)$ \\
\hline 2 & $\mathrm{E}$ & Fluoride & $-14.77(6.3)$ & $-10.71(11.7)$ & $2.18(8.0)$ & $11.08(10.9)$ \\
\hline & $\mathrm{F}$ & No fluoride & $-13.08(6.1)$ & $-11.53(12.6)$ & $3.19(8.8)$ & $2.56(8.3)$ \\
\hline 3 & $\mathrm{G}$ & Fluoride & $-14.27(7.2)$ & $-11.46(14.1)$ & $2.99(7.5)$ & $13.69(10.7)$ \\
\hline & $\mathrm{H}$ & No fluoride & $-13.34(5.5)$ & $-8.14(19.5)$ & $-1.34(14.6)$ & $-3.25(12.6)$ \\
\hline Score & & $0.7988^{\mathrm{a}}$ & $0.1934^{\mathrm{a}}$ & $0.0442^{\mathrm{a}}$ & $0.3963^{\mathrm{a}}$ \\
\hline Fluoride & & $0.7880^{\mathrm{b}}$ & $0.9251^{\mathrm{b}}$ & $0.4284^{\mathrm{b}}$ & $<.0001^{\mathrm{b}}$ \\
\hline Score $\times$ Fluoride & & 0.382 & 0.630 & $0.028^{\mathrm{c}}$ & $<.0001^{\mathrm{c}}$ \\
\hline
\end{tabular}

${ }^{a} p$-value for each factor and interaction between factors.

${ }^{\mathrm{b}} \mathrm{p}$-value for each factor of two predictors group (fluoride and placebo).

'Significant different ( $\mathrm{p}$ value $<0.05$ ) between $\Delta \Delta \mathrm{F}_{10 \mathrm{~d}}, \Delta \Delta \mathrm{F}_{20}$, were found it. 


\section{$\Delta \Delta \mathrm{F} 10 \mathrm{~d}$}

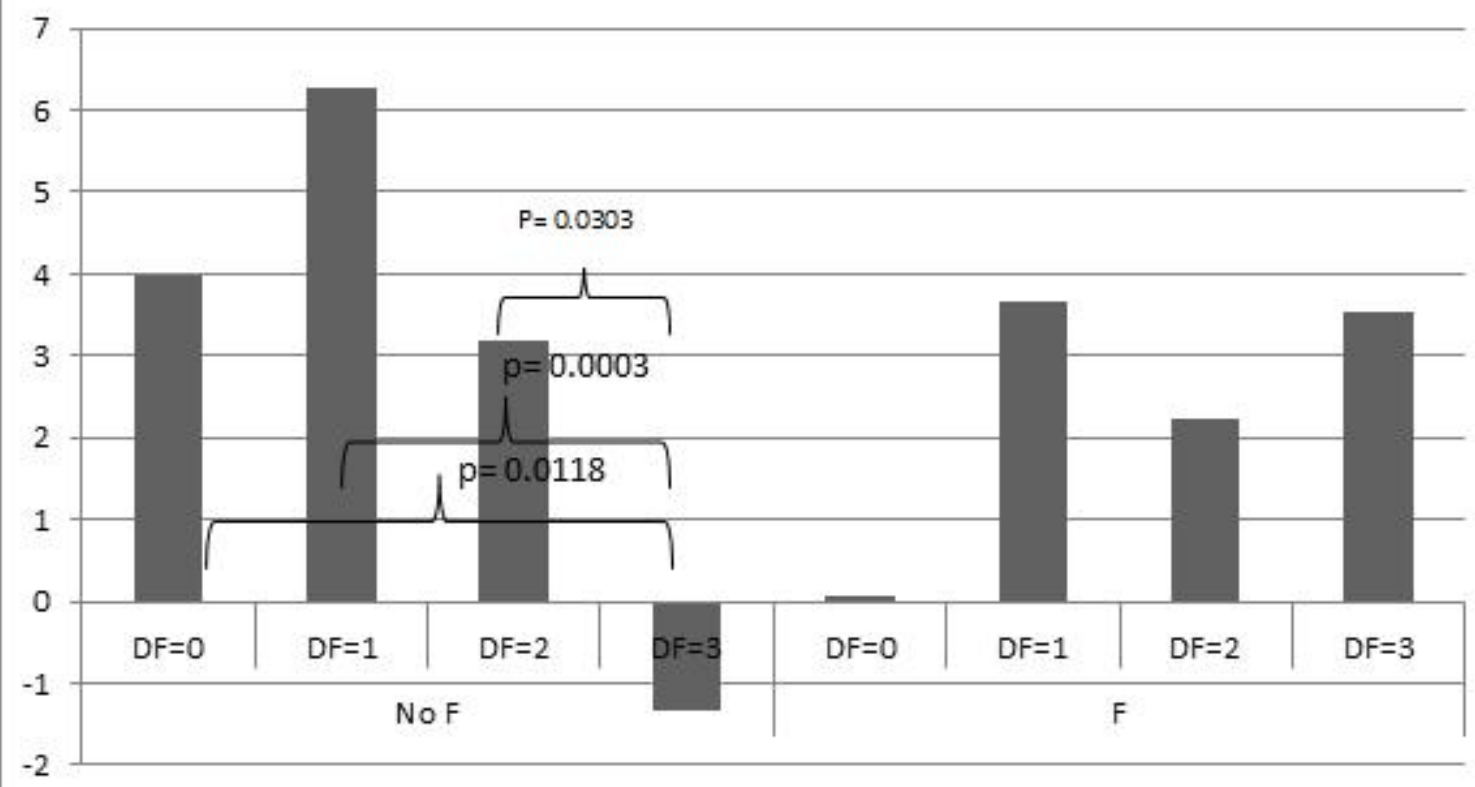

Figure 1. Result of the statistical analysis of the $\Delta \Delta \mathrm{F}_{10 \mathrm{~d}}$ variable for all placebo groups as a function of enamel fluorosis severity 
